\title{
FingerSlide: Investigating Passive Haptic Sliding As A Tacton Channel
}

\author{
Abhijit Karnik \\ Lancaster University \\ Lancaster, UK \\ a.karnik@lancaster.ac.uk \\ Pavels Dembo \\ Lancaster University \\ Lancaster, UK \\ p.dembo@lancaster.ac.uk
}

\author{
Jason Alexander \\ Lancaster University \\ Lancaster, UK \\ j.alexander@lancaster.ac.uk
}

\author{
Kian Meng Yap \\ Sunway University \\ Kuala Lumpur, Malaysia \\ kmyap@sunway.edu.my \\ Hong Jian Wong \\ Sunway University \\ Kuala Lumpur, Malaysia \\ hjwongdr1@gmail.com
}

\begin{abstract}
The haptic sensation of sliding a surface under a probing finger can be used to convey surface information or coded data to the user. In this paper, we investigate users' ability to discern different sliding profiles based on the velocity and direction of sliding for use as haptic-tactons. We built FingerSlide, a novel haptic device which can position and control moving surfaces under a user's finger and used this to run two independent studies. The first study investigates if users can identify the direction of sliding at different velocities. The second study investigates if the users can distinguish a difference between two velocities. Our results show a faster response for higher velocities in the direction study and high error rates in identifying differences in the direction study. We discuss these results and infer design considerations for haptic devices that use the sliding effect to convey information.
\end{abstract}

Keywords-Haptic interfaces; passive sliding; user study;

\section{INTRODUCTION}

Haptic interfaces stimulate the touch and kinesthetic channels of humans to communicate information. The field of haptic interfaces has produced a rich taxonomy of devices that 'touch back' [16] and work as a primary or additional channel of information delivery in a variety of scenarios like medicine [13], mobile interaction [17] and mixed augmented reality (AR) [9]. Devices like the PHANToM [21] or the Novint Falcon support resistive probing and spatial positioning while vibrotactile interfaces like PocketNavigator [27], TeslaTouch [4] and commercial mobile devices support tactile vibration as a communication channel.

Despite this diverse range of research, the potential of device-triggered sliding (or dragging) below a stationary probing finger is underexplored from an interaction perspective. In the real world, when a real object in contact with the finger is subjected to relative motion, the finger experiences passive haptic sensation. Normally, the user performs such an interaction (active probing) with the surface texture or topography. Different underlying physical phenomena can trigger the tactile sensation of sliding; texture coarseness, adhesiveness, relative friction of surface or fluid viscosity, density and flow velocity. While many haptic devices attempt to produce a surface texture that can interface with a moving finger $[4,24]$, we are interested in the passive state of the finger and active sliding of the device surface and use the resulting tactile sensations as metaphor or a means of encoding information.

Psychophysical studies term this form of haptic sensation as part of passive touch but tend to focus on the creation of textures. Passive touch involving sliding can be beneficial in scenarios where the background vibrational noise interferes with the vibrotactile feedback or when a moving finger is impractical (e.g. while driving [2] or when user is wearing protective gloves). A passive touch haptic device can deliver interaction from an extremely small form-factor no wider than the fingertip. On larger scales, a data physicalisation artefact [18] could encode data into the physical behaviour of sliding motion. For example, the download rate of a file transfer or the time remaining can be encoded as a sliding action of a surface relative to a passive probing finger. A sliding tactile button can be envisioned, wherein the response to a click depends on the sliding profile when the button is clicked. In a mobile scenario, a user could check for notifications by interacting with a small sliding surface without viewing the device's display. Similar metaphors have been investigated previously with Gesture Output [29] and SlickFeel [11].

A sliding action consists of the direction of sliding and the velocity with which the surface slides below the finger. A haptic device could encode information in these two parameters. However, it is necessary to understand how well users can distinguish variations in these parameters. In this paper, we attempt to answer this question. For this, we built a prototype called 'FingerSlide' to investigate how well the sliding effect is perceived by users. We ran user studies to see if the users could discern the direction and velocity of sliding. Thus, the contributions of this paper:

- An exploration of the design space of haptic sliding interfaces involving passive touch.

- Two studies that examine users' ability to discern between different sliding profiles.

- Design implications for applications that use haptic sliding to encode information

\section{BACKGROUND}

We propose the use of direction and velocity of sliding to encode information as Tactons [8] and build upon existing studies about sliding interfaces. These studies inform the choice of velocities while existing haptic interfaces inform the design of the prototypes used for study.

\section{A. Haptic Sliding Tactons}

The Tacton framework [8] provides direction on how to generate useful means of coding information. The Tacton framework shows how frequency, amplitude and duration of a tactile pulse act as controllable parameters to encode abstract messages. Pielot et al. [27] demonstrate the use of short and long tactile pulses as structured Tactons for mapping direction in map navigation and also encoding distances [26]. Similarly, velocity and direction of passive 
sliding can support similar abstract message encoding. These can also be combined with the duration of sliding and varying textures. In the current work, we focus on understanding the effects of the first two parameters.

\section{B. Haptic Perception Studies}

Studies investigating how textures can be dynamically generated on surfaces utilize various mechanisms like electrostatic stimulation [4], vibrotactile mechanical pinarrays, textured balls and even ultrasonic-vibration based dynamic friction $[25,34]$. The main focus of these studies is to understand how dynamic surface textures can be generated on a touchscreen which are then probed by a moving finger $[7,23]$. These studies complement proposed models of the human tactile perceptual mechanism. For example, Kuroki et al. [19] propose a triggering model for Meissner corpuscles (RAI type mechanoreceptors) as these are supposed to be more important for active touch than other mechanoreceptors viz. Pacinian corpuscles (RAII), Merkel's discs (SAI) and Ruffini endings (SAII).

Other studies have focused on the perception of passive touch. Bicchi et al. [5] investigates the haptic equivalent of the Barber-pole Illusion [14] which involves the movement of gratings behind a rectangular aperture. Salada et al. [30] conducted two studies about sliding wherein one study investigates if the effect of arm and hand proprioception is higher than the perception of slip on the fingertip. A recent study by Delhaye et al. [12] focuses on modelling surface strain under shearing conditions (which occur during sliding motion). They conclude that there is some dependence on local fingerprint patterns that produces deviation from standard contact models. Biet et al. [6] investigate how different virtual gratings can be perceived by dynamic touch but encourage the movement of the finger to explore the surface.

Psychophysical studies involving passive touch focus on texture. For example, Vega-Bermudez et al. discuss the differences between active and passive touch using raised letters [32]. Lamb [20] investigates texture discrimination for passive touch and finally Meftah et al. [22] investigates textures (roughness of raised dot surfaces). A detailed investigation about the discrimination of velocities magnitudes (speed and direction) independent of the textures is found to be lacking. Meftah et al. state that 'an invariant central representation of surface roughness could be extracted from the ambiguous peripheral signals that covary with roughness and the stimulating conditions (e.g. speed) by means of a simple subtraction process.' These studies build understanding about passive touch related to textures but not about sliding velocities and direction (independent of speed) on a stationary fingertip as haptic interface elements. This motivates our studies and the prototype required for them.

\section{Finger Exploration Velocity}

Finger exploration velocity is the velocity with which an individual moves their fingertip across a surface to explore the surface features. This velocity is independent of the surface properties that enable haptic perception of the surface (as it is controlled by the individual) but is manipulated to achieve discrimination of features. For a haptic sliding surface to work, we need to select sliding velocities that are within this natural range of finger exploration velocities. Different researchers have identified a diverse range of velocities for finger exploration. Salada et al. [30] used a velocity range between $80 \mathrm{~mm} / \mathrm{s}$ to $240 \mathrm{~mm} / \mathrm{s}$ which is informed by Whitsel et al. [33]. Salada states that Whitsel empirically suggests an optimal range of between $30 \mathrm{~mm} / \mathrm{s}$ to $100 \mathrm{~mm} / \mathrm{s}$.

This is in addition to an upper limit around $235 \mathrm{~cm} / \mathrm{s}$ (we believe this to be misreported and should be $235 \mathrm{~mm} / \mathrm{s}$ ). On the other hand, ShiverPaD [10] used $100 \mathrm{~mm} / \mathrm{s}$ as the peak velocity for their apparatus and also found that participants exceeded a finger exploration velocity of $30 \mathrm{~mm} / \mathrm{s}$ in only $0.4 \%$ of their trials. Skedung et al. [31] report their choice of velocity range between $10 \mathrm{~mm} / \mathrm{s}$ and $100 \mathrm{~mm} / \mathrm{s}$ and explain that finer surface features require a lower exploration velocity. We used the observed ranges from ShiverPaD as the velocities for investigation in our studies.

\section{FINGERSLIDE PROTOTYPE}

\section{A. Design Selection}

The main design requirement of a sliding haptic interface is the ability to mimic an infinite planar surface that can move in at least one direction. We looked at existing haptic implementations to identify which were suitable for producing the sliding effect on a stationary finger and evaluate the perception of the sliding effect.

\section{1) LongRangeOuija}

Roudaut et al. [29] used a PHANToM/Falcon to move a slate to 'draw' gestures or numbers. The user's finger rests on the slate and moves along with the slate allowing the user to read the gesture or number drawn by the device. The system shown in Fig. 1a, uses the PHANToM to slide the slate under the user's fingers resulting in passive touch based haptic sliding. However, the slate has a restitution constraint, i.e. it can only travel a small distance in a single direction and needs to reverse and reset to a starting position to continue, limiting its suitability for Fingerslide.

\section{2) HapticEdge}

Jang et al. demonstrated HapticEdge [17] consisting of actuated columns that protrude from the side of a mobile device as shown in Fig. 1b. The height of protrusion is controlled by the attached piezoelectric linear actuators. HapticEdge supports passive touch but does not directly
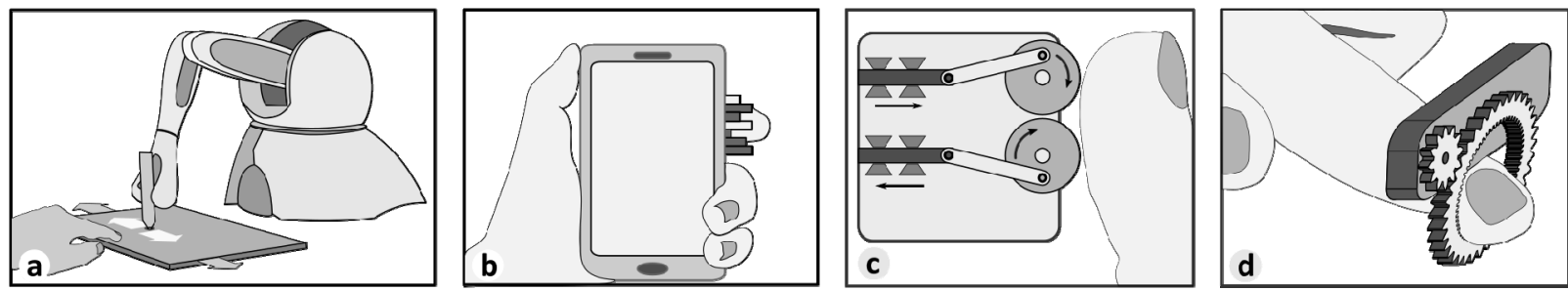

Fig. 1. Prototype Designs: a. longRangeOuija type setup; b. HapticEdge setup; c. Reciprocating cranks design; d. Ring-based sliding surface 

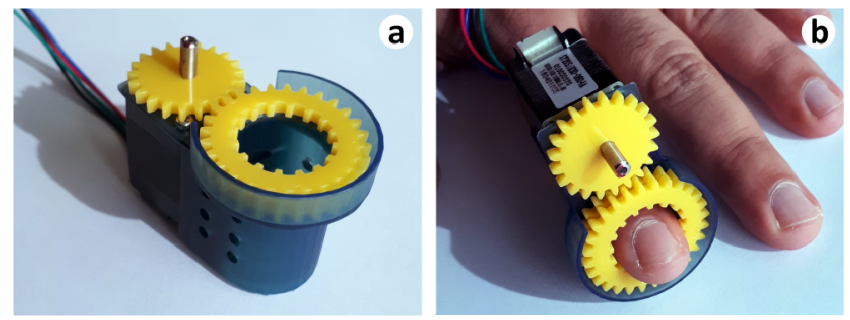

Fig. 3. Ring-based sliding surface prototype.

support sliding motion as it can only generate movement normal to the finger surface. True sliding motion has a shear component (parallel to the surface of the finger) which is absent in HapticEdge. However, it could convey the sliding sensation if the haptic sensory saltation like the 'cutaneous rabbit' effect [15] and spatiotemporal illusions like the tau and kappa effects are employed. From the experimental standpoint, these require separate studies of their own to establish if these work on the fingertip and if they work at the resolution provided by the actuated columns. Thus, this design was considered unsuitable for the experimental prototype in the current context.

\section{3) Reciprocating Cranks Design}

HapticEdge's limitation can be remedied by modifying the design to add an additional crankshaft attachment to the actuated columns. This converts the linear motion of the columns into rotary motion and thus mimics a coarse sliding effect if the probing finger touches the flywheels as shown in Fig. 1c. This design has potential if the linear actuators are able to generate the required force to overcome the friction between the finger and the flywheels and there is more than one flywheel in contact with the probing fingertip. The engineering challenge of building a miniaturized crankflywheel assembly is beyond the scope of this paper.

\section{4) Ring-based Sliding Surface}

This prototype design was partially inspired by the ringshaped receptacles of DesktopGlove [1], HapRing [3] and the reciprocating cranks design. The design (Fig. 1d) is a finger-mounted setup. The sliding surface is the inner surface of spur gear that is worn on the probing fingertip like a ring. A second gear connects the spur gear to a driving motor resting on the fingernail.

The rotating spur gear can mimic a sliding surface and the diameter of the gear determines the contact profile for the finger. A commercial implementation would be likely to take this form as it is similar in form-factor to a haptic glove with eccentric rotating mass (ERM) vibration motors attached to the fingertips. Further texture emulation can be achieved with electrostatic or ultrasonic means as discussed by Meyer et al. [23].

\section{B. Prototype Implementation}

We implemented two versions of the ring-based sliding surface. The first was a finger-mounted design as described above (see Fig. 3). However, for the experiment prototype, we used a more practical and robust implementation. This methodology is similar to Roudaut et al. [29] and decouples engineering challenges of motor selection from the perceptual study requirements. The experimental unit consists of a toothed belt driven by a larger stepper motor. The moving belt acts as the sliding surface when it is in contact with a probing finger. The stepper motor allows the belt to move at a constant and controlled velocity and in two directions. Thus, the belt can emulate a bi-directional sliding surface. The belt and motor are mounted on a hinged platform which can be raised or lowered using a cam attached to a servo (see Fig. 2b, c). The motor is controlled by a PC through an Arduino and a stepper driver. Since the belt has a fixed toothed surface, we lose the fidelity of changing surface textures. However, this was a pragmatic choice as texture emulation is a non-trivial problem and can be tackled in a future study.

\section{EXPERIMENTS}

For haptic sliding to be an effective means of information delivery, first we need to investigate the users' ability to discern different sliding profiles based on the speed and direction of sliding. The first study measures users' ability to identify direction correctly while speed varies. The second study measures the users' ability to distinguish between two velocities differing by a pre-determined magnitude only. We ran a pilot study with three participants to validate the experiment protocol and the velocity ranges. After this, we ran the studies discussed next. We sought the University Ethics Committee's approval before running the studies.

\section{A. Direction Study}

This study aimed to answer the question, "Can users distinguish the direction of sliding correctly and which factors affect this activity?" Our $\mathrm{H}_{0}$ statement was that users would be able to identify the direction of sliding without independent factors like sliding velocity and handedness of the user affecting the outcomes.

\section{1) Task}

The chosen task was a classification task. The participants were asked to place one probing finger (index finger of either hand) on the FingerSlide apparatus. Their task was to identify the direction of travel of the surface and record this information as quickly and accurately as possible.

\section{2) Factors and Measures}

For this study, we recorded the dominant hand (DH vs
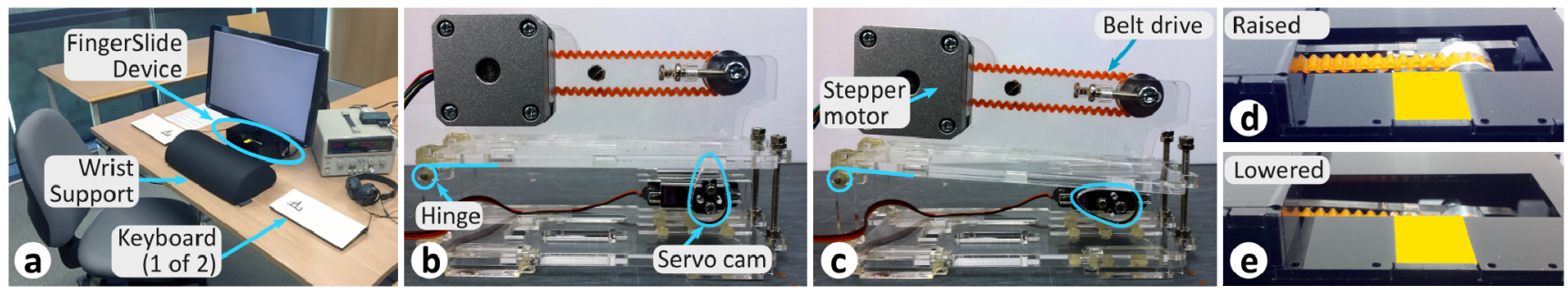

Fig. 2. Apparatus and setup. a. Experimental setup (during experiment, participants could not see the belt as seen in d. and e.); b. Raised state; c. Lowered state with rotated cam; d., e. Raised and lowered view of belt 
non-DH) of the participant to check if this had any effect on the outcomes. Each participant used index fingers from both hands. The participants were instructed to identify the direction of travel of the belt (DIRN: L or R). The other controlled factor was the sliding velocity. As discussed previously, this velocity should lie within the range of comfortable finger exploration velocities. We used the upper velocity value as reported for ShiverPaD [10] and the pilot study to specify the lower velocity value. Thus the direction study's speed range was $10 \mathrm{~mm} / \mathrm{s}$ to $30 \mathrm{~mm} / \mathrm{s}$ and we split this range into 10 equal steps (VEL). We collected metrics to answer the questions "Did the participant correctly identify the direction of travel?" (ACC: Accuracy) and "How long did the participant take to arrive at the answer?" (TCT).

\section{3) Apparatus}

The experiment-version of FingerSlide was used for the study. A $1.8^{\circ}$ step-angle stepper motor drove a $200 \mathrm{~mm}$ long, $6 \mathrm{~mm}$ wide and 50 tooth double-sided V-belt. The teeth on the belt were $2 \mathrm{~mm}$ high and spaced $3.6 \mathrm{~mm}$ apart. The stepper was micro-stepped and maintained the speed of the moving belt within $+/-2 \%$ of the desired value. The complete setup is shown in Fig. 2a. We placed an LCD monitor to display the task progress and instructions. This monitor also hid the movement of the belt from the user's view, thus removing any visual cues to the participant. We also placed two keyboards, one on each side of the device, to facilitate the entry of answers without having to take the finger off the FingerSlide interface. Each keyboard exposed only four keys labelled ' $\mathrm{S}$ ', '<', '>' and '='. The direction study only used the first three, ' $S$ ' for starting the trial, ' $<$ ' for belt sliding left and ' $>$ ' for belt sliding right. We provided a wrist support to prevent fatigue. The stepper motor produced a faintly audible hum. We included noise-cancelling headphones, which played white noise to block the hum from the stepper and prevented users from using the hum to assist with the task.

\section{4) Participants}

We recruited participants from the University campus through email lists. In all, we got 12 participants; 4 females and 8 males aged between $18-45$ years for the study. Only one participant was left-handed and none had prior experience with haptic interfaces apart from mobile devices. The participants were given a $£ 5$ reward for their time.

\section{5) Procedure}

The participants were given time to familiarize themselves with setup and then the task was explained. Once seated, the participant placed one of their index fingers on the apparatus inside the yellow region seen in Fig. 3d. The study consisted of two phases. During the initial phase, consisting of 10 trials, the users familiarized themselves with the experimental procedure. Data from this phase was discarded. Headphones were turned on before starting the next phase during which the participants were asked to perform the task as quickly and accurately as possible.

At the start of every trial, the servo-cam started in the lowered position such that the belt was not in contact with the probing finger. The servo-cam raised the belt into position (touching the participant's finger) when the ' $S$ ' (start) key was pressed on either of the keyboards. Next, the Arduino set the stepper motor's speed and direction. These steps occurred in $<100 \mathrm{~ms}$. The participant then had to click one of the ' $<$ ' or ' $>$ ' keys to register their answer. The program recorded the TCT and ACC for the trial while the belt reset. The next trial would only commence when the participant pressed ' $S$ ' to start it.

The study consisted of 120 trials divided into blocks of 30 trials with a 1-minute break between trials. The trial dataset consisted of a combination of 10 velocity values, 2 directions and 3 repeats per combination for each index finger giving us $(10 \times 2 \times 3 \times 2=) \quad 120$ trials which were randomized and distributed equally across all blocks to minimize order effects. Half of the participants started the first block with their dominant hand (right hand for 11 participants), while the other half started with their nondominant hand. For every subsequent block, they changed hands and placed the other hand's index finger on the apparatus before starting. The entire study took approximately 10 minutes to complete.

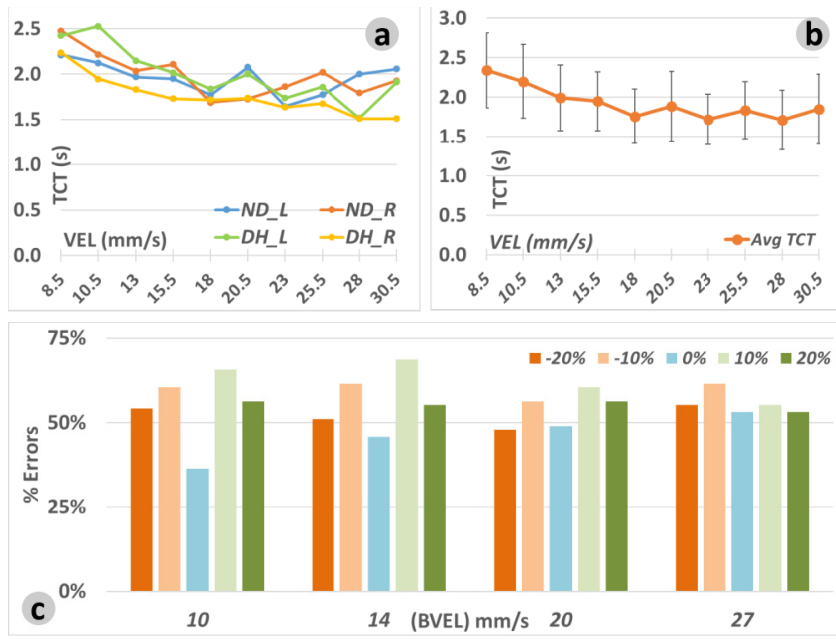

Fig. 4. Analysis of results. Direction Study: a. TCT for $D H \times D I R N$ and VEL (x-axis), b. Avg. TCT. Velocity Study: c. \%age Errors for speed Delta by individual Base speeds (x-axis)

6) Results

We collected data from 1440 trials, which included 1345 correct answers. The experimental design was a withinsubjects repeated measures factorial design $(\mathrm{DH} \times \mathrm{DIRN} \times \mathrm{VEL})$. We ran repeated-measures ANOVA with Bonferroni corrections using SPSS to analyse the results of accuracy and average TCT for repeats. Neither the factors (dominant hand, direction and speed) nor any interaction of these had a statistically significant impact on accuracy. Two participants accounted for 55 errors out of the total 95. The errors distribution did not reveal any features of interest.

The TCT analysis used the trials with correct responses only and revealed observations of interest. There was a significant main effect of speed on TCT, $F_{(2.5,27.7)}=6.25$, $p<0.005$. Analysis of contrasts revealed that TCT was significantly lower for higher speed values as compared to the lowest speed value. None of the other factors or interactions between factors had a significant effect on TCT. Fig. 4a and Fig. 4b show the results. We discuss the implications later in the 'Discussion' section.

\section{B. Velocity Study}

This study aimed to answer the question, "Can users distinguish the difference between two sliding velocities correctly and which factors affect this activity?" Our $\mathrm{H}_{0}$ was that users would be able to identify the difference between 
two velocities differing by a fixed Weber fraction, independently of factors like direction and dominant hand.

\section{1) Factors and Measures}

The participants were instructed to focus on the speed only. The speed range from the direction study was reused. However, instead of 10 steps, only four distinct and equidistant steps were selected as our 'Base Velocities' (BVEL). For each base velocity, another five 'Delta velocities' (DVEL) were chosen as Weber fractions, $+/-20 \%$, $+/-10 \%$ and $0 \%$ offset from the BVEL value. In each trial, the participant experienced a BVEL and one of its matching DVEL in a random and counterbalanced order. We collected metrics to answer the questions "Did participants correctly identify the difference in velocity?" (ACC) and "How long did the participant take to arrive at the answer?" (TCT).

\section{2) Task and Procedure}

A relative difference classification task was chosen as our aim was to check if identification of relative difference between velocities was possible. 'Absolute values' are harder to recall [28] and relative differences modelled as constant fractions allow interpolation based on Weber's Law. Similar to the direction study, participants used their index fingers. They experienced each speed for three seconds with a onesecond gap and had an additional three seconds to answer. The participants were asked to press ' $>$ ' if the second velocity was slower than the first, ' $<$ ' if the second velocity was greater and finally, ' $=$ ' if both were equal. If the participant took more than 6 seconds from the start of the second sliding motion, the trial timed out.

Each participant saw 160 trials (overall 1920 trials), as four blocks of 40 trials, each with a 1-minute break in between. The participants used the same finger for the $\mathrm{n}^{\text {th }}$ block as was used in the direction study. The trials consisted of a combination of BVEL, DVEL, both directions of sliding and two repeats per combination for each finger giving us $(4 \times 5 \times 2 \times 2 \times 2=) 160$ trials for each participant.

TABLE I. CONFUSION MATRIX FOR VELOCITY STUDY

\begin{tabular}{|c|c|c|c|c|c|c|c|c|c|c|}
\hline & \multicolumn{9}{|c|}{ Participants' Answers } \\
\hline & & $E q$ & $\boldsymbol{G r}$ & $S m$ & $E q$ & $G r$ & $S m$ & $E q$ & $G r$ & $S m$ \\
\hline \multirow{3}{*}{ ت } & $E q$ & $54 \%$ & $23 \%$ & $23 \%$ & & & & & & \\
\hline & $G r$ & & & & $40 \%$ & $40 \%$ & $20 \%$ & $34 \%$ & $48 \%$ & $19 \%$ \\
\hline & $S m$ & & & & $40 \%$ & $21 \%$ & $38 \%$ & $35 \%$ & $20 \%$ & $45 \%$ \\
\hline \multicolumn{2}{|c|}{ Weber Fr } & \multicolumn{3}{|c|}{0} & \multicolumn{3}{|c|}{0.1} & \multicolumn{3}{|c|}{0.2} \\
\hline
\end{tabular}

\section{3) Apparatus and Participants}

The apparatus and setup from the direction study was reused for this study. Participants from the direction study took a 5-minute break after it ended and then participated in the velocity study.

\section{4) Results}

We collected data from 1920 trials for analysis. Once again, we planned to run the repeated measures ANOVA analysis similar to the direction study. However, the analysis for ACC revealed a very high number of errors. Five trials timed out, and 1054 trials $(\sim 55 \%)$ had incorrect answers (see Fig. 4c). After eliminating the erroneous trials, no valid cases remained for performing repeated measures ANOVA. Table 1 contains the confusion matrix for the responses versus the ground truth.

\section{DISCUSSION}

\section{A. Experimental Findings}

The two studies provide us with a better understanding of how users are able to distinguish between different sliding profiles. The direction study shows the absence of effect of dominant hand or direction of the sliding action on accuracy or completion time. The primary outcome of the study is that participants can discern the direction faster when the sliding velocity is higher independent of the direction of sliding.

In the velocity study, participants were unable to discern the differences between the presented velocity stimuli. We have insufficient evidence from this study to state conclusively that our stimuli are indistinguishable at the tested velocities. From a psychophysical perspective, this lays the ground for a Just Noticeable Difference study to discern a velocity difference. The current study provides a lower value for the Weber fraction $(>0.2)$ and the current study defines the lower bound for relative speed for tacton features with a defined period. The properties of the belt's surface may prevent differentiation of stimuli at this level contrary to Meftah's observation about independence of textures and velocities. A future study could help correlate the outcomes with the findings of Brewer et al. [7] by looking into viscosity and friction related perception of the surface.

\section{B. Design Implications}

From a design perspective, sliding can be used as a haptic interface in certain cases and we have suggested a few physical design alternatives that can support sliding. The design of such a device is more complicated than vibrotactile and electrostatic haptic signals, which generate forces normal to the surface of the finger. Designs using mechanical actuator should be able to operate without reset or reversal of the sliding surface. Our studies show that a single finger can identify directional features of sufficient duration. Designers can thus construct tactons encoded into direction of sliding, keeping in mind that users can identify direction faster for higher velocities. The studies also show that the ability to discern difference in velocities is not trivial. A bimodal speed tacton (on/off) can be supported but finer resolution requires further studies to identify the exact Weber fraction.

\section{Effect of Texture}

We used a fixed and uniform texture in the form of the double-toothed V-belt in our study. The tooth-profile of the belt resembles a grating pattern used in previous studies [5, 31] and represents a constant base frequency signal that triggers the fingertip mechanoreceptors at $2.8-8.5 \mathrm{~Hz}$ and with fixed maximum amplitude $(2 \mathrm{~mm})$. The actual amplitude is lower as the participants only rest their finger on the belt. Prior research does not report results using a base frequency as the finger exploration velocity is not fixed. Instead, they discuss the wavelength of haptic discrimination. However, in context of haptic sliding interfaces, the frequency can be computed as $f=\lambda \times_{c}$ where $\lambda$, is the distance between successive repeating features of the texture and $\mathrm{c}$, the velocity of the sliding surface. A frequency-based analysis can serve as a baseline for comparing different sliding textures and correlate it to the discrimination frequencies of the fingertip mechanoreceptors in a future study. 


\section{FUTURE WORK}

With FingerSlide, we hope to stimulate discussion about the use of passive touch-based haptic sliding as an interface element. The prototype is limited to a single texture and movement along a single axis. Accommodating 2DOF sliding and variable textures within a small form-factor is a design challenge for future work. Finally, a detailed exploration of tacton design for sliding are directions with open questions and challenges.

\section{CONCLUSION}

In this paper, we have discussed the use of haptic sliding as a means of communicating information. We discussed designs that support haptic sliding interfaces. We further explored the ability of users to identify different sliding profiles based on velocity and direction using the experiment apparatus 'FingerSlide'. We ran two studies, which showed that users discern the direction tacton faster if the sliding velocity is higher. We also found out that the JND threshold for relative velocity comparison is larger than 0.2 . We proposed design recommendations based on these findings.

\section{ACKNOWLEDGMENT}

This work was funded through the Lancaster UniversitySunway University Internal Grant Scheme.

\section{REFERENCES}

[1] M. Achibet, G. Casiez and M. Marchai. DesktopGlove: A multifinger force feedback interface separating degrees of freedom between hands. in IEEE Symposium on 3DUI, 2016, pp. 3-12.

[2] M. Ahtamad, C. Spence, C. Ho and R. Gray. Warning Drivers about Impending Collisions Using Vibrotactile Flow. IEEE Trans. Haptics, 9 (1). 2016, pp. 134-141.

[3] O. Ariza, P. Lubos, F. Steinicke and G. Bruder. Ring-shaped haptic device with vibrotactile feedback patterns to support natural spatial interaction Proc. 25th International Conference on Artificial Reality and Telexistence. 2015, pp. 175-181.

[4] Olivier Bau, Ivan Poupyrev, Ali Israr and Chris Harrison. TeslaTouch: Electrovibration for touch surfaces Proc. of UIST 2010, ACM, 2010, pp 283-292.

[5] Antonio Bicchi, Enzo P. Scilingo, Emiliano Ricciardi and Pietro Pietrini. 2008. Tactile flow explains haptic counterparts of common visual illusions. Brain Research Bulletin, 75 (6). pp. 737-741. http://dx.doi.org/10.1016/j.brainresbull.2008.01.011

[6] M. Biet, G. Casiez, F. Giraud and B. Lemaire-Semail. Discrimination of Virtual Square Gratings by Dynamic Touch on Friction Based Tactile Displays. Symposium on Haptic Interfaces for Virtual Environment and Teleoperator Systems. 2008, pp. 41-48.

[7] D. J. Brewer, D. J. Meyer, M. A. Peshkin and J. E. Colgate. Viscous textures: Velocity dependence in fingertip-surface scanning interaction. IEEE Haptics Symposium, 2016, pp. 265-270.

[8] Stephen Brewster and Lorna M. Brown. Tactons: structured tactile messages for non-visual information display Proc. $5^{\text {th }}$ Conference on Australasian User Interface. 2004, pp. 15-23.

[9] Volkert Buchmann, Stephen Violich, Mark Billinghurst and Andy Cockburn. FingARtips: gesture based direct manipulation in AR. Proc. of GRAPHITE '04, ACM, 2004, pp. 212-221.

[10] E. C. Chubb, J. E. Colgate and M. A. Peshkin. ShiverPaD: A Glass Haptic Surface That Produces Shear Force on a Bare Finger. IEEE Trans on Haptics, 3 (3), 2010, pp. 189-198.

[11] Xiaowei Dai, Jiawei Gu, Xiang Cao, J. Edward Colgate and Hong Tan. SlickFeel: sliding and clicking haptic feedback on a touchscreen Adjunct Proc. UIST' 12, ACM, 2012, pp. 21-22.
[12] Benoit Delhaye, Allan Barrea, Benoni B. Edin, Philippe Lefèvre and Jean-Louis Thonnard. Surface strain measurements of fingertip skin under shearing. J. Royal Society Interface, 13 (115), 2016.

[13] David Escobar-Castillejos, Julieta Noguez, Luis Neri, Alejandra Magana and Bedrich Benes. A Review of Simulators with Haptic Devices Medical Training. J. Medical Systems, 40 (4). pp. 1-22.

[14] Nick Fisher and Johannes M Zanker. The Directional Tuning of the Barber-Pole Illusion. J. Perception, 30 (11), 2001, pp. 1321-1336.

[15] Frank A. Geldard and Carl E. Sherrick. The Cutaneous "Rabbit": A Perceptual Illusion. Science, 178 (4057), 1972, pp. 178-179.

[16] Vincent Hayward, Oliver R. Astley, Manuel Cruz-Hernandez, Danny Grant and Gabriel Robles-De-La-Torre. Haptic interfaces and devices. Sensor Review, 24 (1), 2004, pp. 16-29.

[17] Sungjune Jang, Lawrence H. Kim, Kesler Tanner, Hiroshi Ishii and Sean Follmer. Haptic Edge Display for Mobile Tactile Interaction Proc. CHI'16, ACM, 2016, pp. 3706-3716.

[18] Yvonne Jansen, Pierre Dragicevic, Petra Isenberg, Jason Alexander, Abhijit Karnik, Johan Kildal, Sriram Subramanian and Kasper Hornbæk. Opportunities and Challenges for Data Physicalization Proc. CHI'15, ACM, 2015, pp. 3227-3236.

[19] Shinobu Kuroki, Hiroyuki Kajimoto, Hideaki Nii, Naoki Kawakami and Susumu Tachi. Proposal of the Stretch Detection Hypothesis of the Meissner Corpuscle. EuroHaptics, Springer, 2008, pp. 245-254.

[20] G. D. Lamb. Tactile discrimination of textured surfaces: psychophysical performance measurements in humans. J. Physiology, 338 (1), 1973, pp. 551-565.

[21] Thomas H Massie and J Kenneth Salisbury. The phantom haptic interface: A device for probing virtual objects. Proc. ASME Winter Annual Meeting. 1994, pp. 295-300.

[22] El Mehdi Meftah, Loïc Belingard and C. Elaine Chapman. Relative effects of the spatial and temporal characteristics of scanned surfaces on human perception of tactile roughness using passive touch. Experimental Brain Research, 132 (3), 2000, pp. 351-361.

[23] D. J. Meyer, M. Wiertlewski, M. A. Peshkin and J. E. Colgate. Dynamics of ultrasonic and electrostatic friction modulation for rendering texture on haptic surfaces. in IEEE Haptics Symposium, 2014, pp. 63-67.

[24] Joe Mullenbach, Craig Shultz, J. Edward Colgate and Anne Marie Piper. Exploring affective communication through variable-friction surface haptics Proc. CHI'14, ACM, 2014, pp. 3963-3972.

[25] Joe Mullenbach, Craig Shultz, Anne Marie Piper, Michael Peshkin and J Edward Colgate. TPad Fire: Surface Haptic Tablet.

[26] Martin Pielot, Oliver Krull and Susanne Boll. Where is my team: supporting situation awareness with tactile displays. Proc. CHI'10, ACM, 2010, pp. 1705-1714.

[27] Martin Pielot, Benjamin Poppinga and Susanne Boll. PocketNavigator: vibro-tactile waypoint navigation for everyday mobile devices Proc. MobileHCI'10, ACM, 2010, pp. 423-426.

[28] Hayne W Reese. 2013. Relational Theories: Configuration Theory. in The perception of stimulus relations: Discrimination learning and transposition, Academic Press, 239.

[29] Anne Roudaut, Andreas Rau, Christoph Sterz, Max Plauth, Pedro Lopes and Patrick Baudisch. Gesture output: eyes-free output using a force feedback touch surface. Proc. CHI'13, ACM, 2013, pp. 2547-56

[30] M. Salada, P. Vishton, J. E. Colgate and E. Frankel. Two experiments on the perception of slip at the fingertip. HAPTICS '04, pp. 146-153.

[31] Lisa Skedung, Martin Arvidsson, Jun Young Chung, Christopher M. Stafford, Birgitta Berglund and Mark W. Rutland. Feeling Small: Exploring the Tactile Perception Limits. Scientific Reports, 3. 2617.

[32] F. Vega-Bermudez, K. O. Johnson and S. S. Hsiao. Human tactile pattern recognition: Active versus passive touch, velocity effects, and patterns of confusion. J. Neurophysiology, 65 (3). pp. 531-46.

[33] B. L. Whitsel, D. A. Dreyer, M. Hollins and M. G. Young. The Coding of Direction of Tactile Stimulus Movement: Correlative Psychophysical and Electrophysiological Data. Sensory Functions of the Skin of Humans, Springer US, Boston, MA, pp. 79-107.

[34] Laura Winfield, John Glassmire, J Edward Colgate and Michael Peshkin. T-pad: Tactile pattern display through variable friction reduction. Proc. Of WHC'07, IEEE, 2007, pp. 421-426. 